\title{
Enzalutamide Associated with Stevens-Johnson Syndrome/Toxic Epidermal Necrolysis (SJS/TEN) Overlap: A Case Report
}

\author{
Sharjeel Israr'1, Christopher R. Mellon², Hayley J. Springs ${ }^{3}$, Asia N. Quan ${ }^{4}$, Marc R. Matthews ${ }^{*}$ \\ ${ }^{1}$ Department of General Surgery, Creighton University School of Medicine, Phoenix Campus, St. Joseph Hospital and Medical \\ Center, Phoenix, AZ, USA \\ ${ }^{2}$ Honor Health Medical Center, Department of Surgery, Scottsdale, AZ, USA \\ ${ }^{3}$ Department of General Surgery, Honor Health John C. Lincoln Medical Center, Phoenix, AZ, USA \\ ${ }^{4}$ Department of Pharmacy, Valleywise Health Medical Center, Phoenix, AZ, USA \\ ${ }^{5}$ The Arizona Burn Center, Phoenix, AZ, USA \\ Email: sharjeelisrar@creighton.edu,crmellon@mac.com, Hayleysprings@gmail.com, \\ asia.quan@valleywisehealth.org, ‘marc_matthews@dmgaz.org
}

How to cite this paper: Israr, S., Mellon, C.R., Springs, H.J., Quan, A.N. and Matthews, M.R. (2021) Enzalutamide Associated with Stevens-Johnson Syndrome/Toxic Epidermal Necrolysis (SJS/TEN) Overlap: A Case Report. Surgical Science, 12, 421-426. https://doi.org/10.4236/ss.2021.1212045

Received: November 23, 2021

Accepted: December 28, 2021

Published: December 31, 2021

Copyright $\odot 2021$ by author(s) and Scientific Research Publishing Inc. This work is licensed under the Creative Commons Attribution International License (CC BY 4.0).

http://creativecommons.org/licenses/by/4.0/

\begin{abstract}
Enzalutamide is a hormonal therapy that blocks the action of androgens, such as testosterone in the treatment of metastatic castration-resistant prostate cancer. Stevens-Johnson syndrome and toxic epidermal necrolysis (SJS/TEN) overlap and are part of an adverse drug reaction continuum of disease, in which there is a $10 \%-30 \%$ involvement of the skin surface with mucositis, blisters, skin slough, and a macular rash. A 66-year-old male was treated with enzalutamide for metastatic prostate cancer and developed SJS/TEN overlap with $25 \%$ total body surface area skin involvement. The patient received a seven-day course of cyclosporine to which he responded by re-epithelialization but succumbed to multi-organ failure. While SJS/TEN has been reported with apalutamide, to our knowledge, this is the first case of SJS/TEN overlap with enzalutamide.
\end{abstract}

\section{Keywords}

Prostate Cancer, Enzalutamide, Stevens-Johnson Syndrome, Toxic Epidermal Necrolysis, SJS/TEN

\section{Introduction}

Enzalutamide is a hormonal therapy that blocks the action of androgens, such as testosterone in the treatment of castration-resistant metastatic prostate disease 
[1] [2] [3]. Among its side effects are hot flashes, impaired sexual function, loss of desire for sex, diarrhea, nausea, itching, weakened bones, seizures, cardiac, vascular and psychiatric disorders [1] [2] [3]. Other examples of this type of androgen inhibitor hormonal therapy are flutamide, bicalutamide, enzalutamide, apalutamide, and nilutamide [1]. Stevens-Johnson syndrome and toxic epidermal necrolysis (SJS/TEN) overlap is part of an adverse drug reaction continuum of disease, in which there is mucositis and a $10 \%-30 \%$ involvement of the skin surface, in which there are blisters, macular papules, skin slough, and a positive Nikolsky sign [4] [5]. Mortality for patients with SJS/TEN is approximately $30 \%$ and increases with advanced age and comorbidities; the major issues of concern are sepsis, infections and multiple organ failure [5] [6]. This manuscript describes an elderly male with metastatic prostate cancer who developed SJS/TEN as a result of enzalutamide therapy and subsequently died as a result of multiple complications and multisystem organ failure. This case report was approved by the Valleywise Institutional Review Board (\#CR2021-020).

\section{Case Report}

A 66-year-old man developed SJS/TEN (25\% total body surface area) one month after receiving enzalutamide (Pfizer, New York, NY, and Astellas Pharma, Tokyo, Japan) (160 mg daily) for his castration-resistant stage IV prostate cancer with lung and intrathoracic lymph node metastases. Taking no other medications but feeling fatigued, he developed a macular rash on his face, scalp, and trunk six days before transfer to the burn center (Figure 1). Transfer to our burn center was immediately initiated for the skin rash and threatened loss of airway with oral mucosal hemorrhage and bleeding, which required an emergency intubation. He had erythematous sloughing of the skin over the chest, back, penis, gluteal cleft and a macular rash on the trunk and all extremities at the time of transfer. Besides prostate cancer, his major comorbidities were chronic obstructive

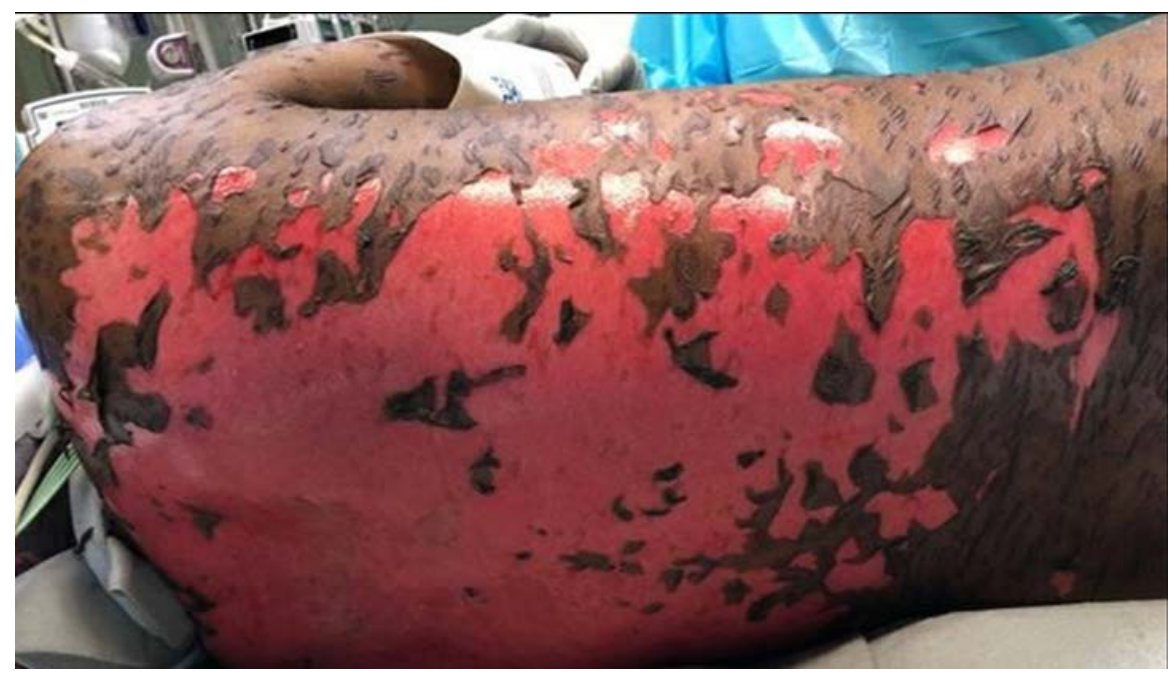

Figure 1. Photograph of patient's back and right flank pre-debridement with epidermal/dermal sloughing consistent with SJS/TEN. 
pulmonary disease, emphysema, hypertension, hypothyroidism, erectile dysfunction, and glaucoma. The admission vital signs were a blood pressure of $86 / 53 \mathrm{mmHg}$, heart rate 82 beats per minute, respirations 14 breaths per minute, temperature $97.7^{\circ}$ Fahrenheit. Admission laboratory blood analysis was obtained (Table 1). By the SCORTEN (Score of Toxic Epidermal Necrolysis) [7], he received 1 point each for $>40$-years-old, associated malignancy, serum BUN $>28$ $\mathrm{mg} / \mathrm{dl}$, $>10 \%$ detached skin surface, and $\mathrm{HCO}_{3}<20 \mathrm{mEq} / \mathrm{L}$, which placed him at $>90 \%$ risk for mortality.

Enzalutamide was discontinued and intravenous cyclosporine at $4 \mathrm{mg} / \mathrm{kg}$ was initiated for a seven-day course [8]. Supportive care measures were implemented, including fluid resuscitation, post-pyloric feeding tube placement for nutrition, deep venous thrombosis chemoprophylaxis, and complication management. The wound dressings included, Mepilex Ag (Mőlnycke, Sweden), Coban on the trunk, Xeroform (3\% bismuth tribromophenate petroleum) dressing (Medline Industries, Inc., Northfield, IN) with bacitracin (Xellia Pharmaceuticals, Chicago, IL) on the face and scalp. Within two weeks, the wounds re-epithelialized.

The patient's hospital course was complicated by prolonged intubation, eventually requiring tracheostomy tube placement. The patient also developed ventilator-associated pneumonias, parapneumonic effusions, and then loculated empyemas requiring two surgical thoracic decortications. In addition, he developed multiple episodes of sepsis and urinary tract infections that required treatment with intravenous antibiotics for Staphylococcus aureus and Klebsiella oxytoca, as well as antifungal intravenous therapy for Candida parapsilos. Finally, with the development of hemorrhagic cystitis, he underwent bladder irrigation therapy which led to a bladder perforation. Subsequent exploratory laparotomy and bladder repair ensued, however, the family elected to withdraw support and the patient succumbed to sepsis and multisystem organ failure after 59-days of hospitalization.

Table 1. Admission Laboratory Blood Values (with reference ranges).

\begin{tabular}{cc}
\hline White blood count (WBC) & $6.4\left(4.5-11 \times 10^{9} / \mathrm{L}\right)$ \\
Hemoglobin & $10.6(13.5-17.5 \mathrm{~g} / \mathrm{dL})$ \\
Platelets & $06(150-450 / \mathrm{microliter})$ \\
Glucose & $113(82-115 \mathrm{mg} / \mathrm{dl})$ \\
Thyroid Stimulating Hormone (TSH) & $4.77(0.50-8.90 \mathrm{uIU} / \mathrm{ml})$ \\
Blood urea nitrogen & $39(8-23 \mathrm{mg} / \mathrm{dl})$ \\
Creatinine & $0.93 \mathrm{mg} / \mathrm{dl}(0.66-1.25)$ \\
pH & $7.292(7.35-7.45)$ \\
HCO 3 & $19.5(22.0-26.0 \mathrm{mmol} / \mathrm{L})$ \\
C-Reactive Protein $(\mathrm{CRP})$ & $308.6(\mathrm{high}>3.0 \mathrm{mg} / \mathrm{L})$. \\
\hline
\end{tabular}




\section{Discussion}

Although rare, there have been adverse skin drug reactions and rashes with androgen receptor inhibitors. The incidence of SJS/TEN in the general population has been reported as 2 cases per million people/year and mortality as high as 50\% [9]. The 2016 Australian Public Assessment Report for enzalutamide indicated an overall incidence of any adverse skin reaction in 871 patients receiving enzalutamide to be slightly higher $169(19.4 \%)$ than in 844 patients receiving placebo 115 (13.5\%) [2]. A comment noted that of two patients with maculopapular rashes, one was of concern: “... The narrative for another patient describes the presence of an erythematous maculopapular rash, with two small pustules and skin peeling of $<20 \%$ total body surface area, but Nikolsky sign negative. The skin biopsy was reported to be 'not inconsistent with a diagnosis of toxidermia.' In both patients, their symptoms abated following temporary discontinuation of enzalutamide ...." [2]

There have been two additional reports in the literature of skin adverse reactions due to enzalutamide medication: acute generalized exanthematous pustulosis (AGEP) in a 62-year-old male on the back and extremities [10]; a diffuse erythematous maculopapular skin eruption on the trunk and extremities (not photo-distributed) in an 87-year-old male [11]. Saito-Sasaki, et al., also provided references for six cases of adverse skin reactions (photosensitivity and pseudoporphyria) attributed to the androgen receptor inhibitor, flutamide [11].

Two fatal cases of TEN have been ascribed to apalutamide, also an androgen-receptor signaling inhibitor in hormonal therapy of castration-resistant metastatic Stage IV prostate cancer [12] [13]. A 77-year-old Japanese male patient with prostate cancer metastases to the bladder and rectum (on $240 \mathrm{mg}$ daily of apalutamide) developed erythema, atypical targetoid lesions over 70\% TBSA, mucositis, liver and renal dysfunction (SCORTEN score 6) [12]. Apalutamide and other medications were stopped; a lymphocyte transformation test and skin biopsy suggested apalutamide as the adverse drug reaction-TEN. He received methylprednisolone pulse therapy $(1000 \mathrm{mg} / \mathrm{day}$ for three days and intravenous immunoglobulin therapy ( $400 \mathrm{mg} / \mathrm{kg} /$ day for 5 days). The skin slough continued, additional lymph node and adrenal metastases developed, and the patient died 53-days after admission from multi-organ failure [12].

Endo, et al., described an 83-year-old Japanese male patient with multiple comorbidities: COPD, cardiac insufficiency, HTN, myelodysplastic syndrome and dementia, who developed a reddish maculopapular rash six weeks after initiating apalutamide $240 \mathrm{mg}$ daily [13]. The patient had a positive Nikolsky sign with atypical targetoid lesions and exfoliation; his SCORTEN score was 4 [13]. He received methylprednisolone pulse therapy, intravenous gamma globulin (400 mg/kg for 5 days) and 7 days of plasmapheresis, but developed a bacterial pneumonia and died 36 days after admission [13]. Kawakami, et al., describe a 78-year-old Japanese male patient with HTN, hyperlipidemia and reflux esophagitis, who developed a generalized pruritic rash after receiving $240 \mathrm{mg}$ apaluta- 
mide daily [14]. The rash was a confluent scaly erythema on the legs with urticaria-like erythematous lesions over 70\% TBSA on the arms and trunk. He also developed a peripheral eosinophilia of $21.6 \%$. There was no mucositis, liver dysfunction, Nikolsky sign or target lesions; the biopsy showed spongiotic dermatitis with no apoptotic keratinocytes but lymphocytes and eosinophilic perivascular infiltration in the upper dermis. The drug was stopped; he responded to prednisolone and apalutamide was restarted at $180 \mathrm{mg}$ /day with prednisolone 1 $\mathrm{mg} /$ day. The drug was continued at $120 \mathrm{mg} /$ day with an increase of prednisolone to $5 \mathrm{mg} /$ day. Within a week, the lesions improved, the prednisolone was tapered, and the patient did well. A Janssen MD Professional Information Resource on apalutamide noted that the incidence of a rash with apalutamide might be more common in the Japanese population due to dosage or genetics but that is only conjecture and unproven [15].

In this case of SJS/TEN caused by enzalutamide, the patient had an elevated SCORTEN placing him at $90 \%$ mortality risk. The patient was not taking any other medication, so in retrospect, the only possible offending medication that might have caused SJS/TEN was enzalutamide. The cascade of clinical problems that developed from pneumonias, sepsis, the need for two thoracic decortications for empyemas and finally his exploratory laparotomy to repair a ruptured bladder could have possibly been avoided if he had not initially received enzalutamide.

\section{Conclusion}

While TEN has been reported with apalutamide, to our knowledge, this is the first case of SJS/TEN overlap with enzalutamide. Additional reported cases in the future will be important to determine the risks for the use of the androgen signaling inhibitors in the development of adverse skin reactions such as SJS/TEN in patients with metastatic prostate cancer. Usage may require modulation of the dosage and type of hormonal therapy dependent on the patient age as less or greater than 65-year-old, comorbidities such as hypertension, ethnicity, and others.

\section{Conflicts of Interest}

The authors declare no conflicts of interest regarding the publication of this paper.

\section{References}

[1] NIH National Cancer Institute (2021) Enzalutamide. https://www.cancer.gov/about-cancer/treatment/drugs/enzalutamide

[2] Australian Public Assessment Report for Enzalutamide. May 2016. https://www.tga.gov.au/sites/default/files/auspar-enzalutamide-160323.pdf

[3] Adverse Reactions in Metastatic CRPC: Prevail Trial. https://www.xtandihcp.com/safety/metastatic-crpc/prevail 
[4] Grünwald, P., Mockenhaupt, M., Panzer, R. and Emmert, S. (2020) Erythema Multiforme, Stevens-Johnson Syndrome/Toxic Epidermal Necrolysis-Diagnosis and Treatment. Journal of the German Society of Dermatology, 18, 547-553. https://doi.org/10.1111/ddg.14118

[5] Charlton, O.A., Harris, V., Phan, K., Mewton, E., Jackson, C. and Cooper, A. (2020) Toxic Epidermal Necrolysis and Stevens-Johnson Syndrome: A Comprehensive Review. Advances Wound Care, 9, 426-439. https://doi.org/10.1089/wound.2019.0977

[6] Lerch, M., Mainetti, C., Beretta-Piccoli, B.T. and Harr, T. (2018) Current Perspectives on Stevens-Johnson Syndrome and Toxic Epidermal Necrolysis. Clinical Reviews in Allergy \& Immunology, 54, 147-176.

https://doi.org/10.1007/s12016-017-8654-Z

[7] Bastuji-Garin, S., Fouchard, N., Bertocchi, M., et al. (2000) SCORTEN: A Severity-of-Illness Score for Toxic Epidermal Necrolysis. Journal of Investigative Dermatology, 115, 149-153. https://doi.org/10.1046/j.1523-1747.2000.00061.x

[8] Gilbert, M. and Scherrer, L.A. (2019) Efficacy and Safety of Cyclosporine in Stevens-Johnson Syndrome and Toxic Epidermal Necrolysis. Dermatologic Therapy, 32, e12758. https://doi.org/10.1111/dth.12758

[9] Letko, E., Papaliodis, D.N., Papaliodis, G.N., Daoud, Y.J., Ahmed, A.R. and Foster, C.S. (2005) Stevens-Johnson Syndrome and Toxic Epidermal Necrolysis: A Review of the Literature. Annals of Allergy, Asthma \& Immunology, 94, 419-436. https://doi.org/10.1016/S1081-1206(10)61112-X

[10] Alberto, C., Konstantinou, M.P., Martinage, C., et al. (2016) Enzalutamide Induced Acute Generalized Exanthematous Pustulosis. Journal of Dermatological Case Reports, 10, 35-38. https://doi.org/10.3315/jdcr.2016.1226

[11] Saito-Sasaki, Sawada, Y., Okado, E. and Nakamura, M. (2018) Drug Eruption Caused by Enzalutamide: A Case and Literature Review of Androgen Receptor-Related Drug Eruptions. Australasian Journal of Dermatology, 59, e133-e134. https://doi.org/10.1111/ajd.12694

[12] Sagawa, N., Watanabe, Y., Mizuno, Y., et al. (2020) A Case of Toxic Epidermal Necrolysis Associated with Apalutamide Administration. Journal of Cutaneous Immunology and Allergy, 3, 134-135. https://doi.org/10.1002/cia2.12138

[13] Endo, Y., Oka, A., Uehara, A., et al. (2020) Fatal Case of Toxic Epidermal Necrolysis Due to Apalutamide Used as a Novel Prostate Cancer Drug. The Journal of Dermatology, 47, e359-e360. https://doi.org/10.1111/1346-8138.15510

[14] Kawakami, Y., Mitsul, M., Takamoto, H. and Yamamoto, Y. (2021) Apalutamide-Induced Exanthematous Drug Eruption Displaying Spongiotic Dermatitis Successfully Treated with Dose Reduction. International Journal of Dermatology, 60, e315-e317. https://doi.org/10.1111/ijd.15420

[15] Janssenpharmaceutical K.K. (2019) Interview from Erleada Tablet 40 mg. 4th. https://www.janssenmd.com/erleada/safety/skin-reaction/erleada-stevensjohnson-s yndrometoxic-epidermal-necrolysis 